particularly nausea. There were no significant effects on clinical laboratory tests, vital signs, ECG or physical examination.

Multiple doses of MBS2320 (75 mg) were also generally well tolerated by subjects with RA when co-administered with MTX. There were no clinically significant findings or trends in the laboratory tests, vital signs or ECG data, and no clinically significant findings on physical examination. No SAEs were reported. The majority of TEAEs were mild in severity with diarrhoea and nausea reported most frequently.

In healthy subjects, MBS2320 was steadily absorbed following doses of 50 to $375 \mathrm{mg} \mathrm{MBS2320.} \mathrm{Systemic} \mathrm{exposure} \mathrm{(AUC)} \mathrm{increased} \mathrm{in} \mathrm{a} \mathrm{dose-}$ proportional manner across all doses and was unaffected by food. In RA patients there was no indication of a PK interaction between MTX and MBS2320 in either direction.

In patients with RA, serum CTX-1, P1NP and osteocalcin increased following 14 days of treatment, while TRAP5b was decreased or unchanged. Median CRP was decreased relative to baseline in after 7 days' treatment with MBS2320, and remained low on Day 14.

Conclusion: MBS2320 was well tolerated for up to 14 days when administered to healthy volunteers and to patients with RA receiving MTX. Systemic exposure was dose-proportional with no evidence of a pharmacokinetic interaction with MTX. Evaluation of biomarkers of bone turnover and inflammation in RA patients showed changes consistent with those expected of an agent with the potential to directly protect the bone and simultaneously ameliorate inflammation.

Louise Jopling, Ian Anderson, Ian Gourley, Helen Marley and Ezi Otti Disclosure of Interests: Lisa Patel Shareholder of: Shareholder of Istesso Ltd, Employee of: Employee of Istesso, Paul Marcus Consultant for: Istesso Ltd, Jim Bush Shareholder of: Labcorp, Employee of: Covance, Andy Gray Shareholder of: Shareholder of Istesso Ltd, Consultant for: Istesso Ltd, Employee of: Astra Zeneca, Richard Fitzgerald: None declared, Sonya Abraham Shareholder of: UCB Pharma, Grant/research support from: Pfizer, Riche, Abbvie, Consultant for: Abbvie Roche Pfizer BMS UCB pharma, Eli Lilly, Employee of: UCB Pharma, Martyn Foster Shareholder of: AstraZeneca, Consultant for: Istesso, Levicept, Employee of: AstraZeneca, Hiep Huatan Consultant for: Istesso Ltd, Employee of: Pfizer, Laurence Skillern Consultant for: Istesso, Employee of: Astellas, Anna Daroszewska Consultant for: Istesso Ltd, Rob van't Hof Shareholder of: OsteoRx Ltd, Sam Williams Shareholder of: Shareholders of Istesso Ltd, Employee of: Employees of Istesso

DOI: 10.1136/annrheumdis-2019-eular.2794

\section{THU0187 EFFICIENCY OF TREATMENT SEQUENCES CONTAINING TOFACITINIB FOR RHEUMATOID ARTHRITIS IN SPAIN}

Carmen Peral ${ }^{1}$, Alejandro Balsa ${ }^{2}$, Federico Navarro ${ }^{3}$, José Luis Pablos ${ }^{4}$, Jose Manuel Martinez Sesmero ${ }^{5}$, Susana Gómez ${ }^{1}$, Monica Valderrama ${ }^{1}$, Itziar Oyagüez ${ }^{6}$, Miguel Angel Casado ${ }^{6}$, Fernando De Andres ${ }^{6}$, Maria Montoro ${ }^{1}$. ${ }^{1}$ Pfizer, Madrid, Spain; ${ }^{2}$ Hospital La Paz, Madrid, Spain; ${ }^{3}$ Hospital QuironSalud Infanta Luisa, Sevilla, Spain; ${ }^{4}$ Hospital Universitario 12 de Octubre, Madrid, Spain; ${ }^{5}$ Hospital Universitario Clínico San Carlos, Madrid, Spain; ${ }^{6}$ PORIB, Madrid, Spain

Background: The availability of oral janus kinase inhibitors, as tofacitinib, has extended the treatment pathways for management of patients with rheumatoid arthritis (RA)

Objectives: To assess the cost-effectiveness of tofacitinib as second-line treatment compared to treatment sequences containing standard biologicaltherapies in patients with moderate to severe RA after failure to diseasemodifying antirheumatic drugs (DMARDs) from the Spanish National Health System perspective.

Methods: A patient-level microsimulation model was used to compare the lifetime cost and quality-adjusted life-years (QALY) for treatment sequences initiating with tofacitinib (5mg BID) followed by biological therapies versus sequences of biological treatments excluding tofacitinib. The sequences were selected by a panel of experts based on clinical practice in Spain. Concomitant treatment with methotrexate (MTX) was considered along all the therapies in the treatment sequences.

Model parameters included age, weight, initial Health Assessment Questionnaire $(\mathrm{HAQ})$ score and clinical response to short and long treatment. Efficacy was measured by means of $\mathrm{HAQ}$ score changes using mixedtreatment-comparisons (for the first 6 months) and data from long-term extension trials (for later periods).

Serious adverse event (SAE) information derived from literature.

The estimation of total cost for sequences included: drugs acquisition (public ex-factory prices with mandatory deduction or reference prices) and parenteral administration, disease progression and SAE management.
Local unitary costs $(€, 2018)$ were applied. Additional comparisons were explored testing other potential sequences.

Results: The base case results showed that sequences initiating with tofacitinib provided greater outcomes than the correspondent sequences excluding tofacitinib. In scenario 1 tofacitinib $+M T X \rightarrow$ Rituximab+MTX $\rightarrow$ sc Tocilizumab+MTX $\rightarrow$ Etanercept + MTX $\rightarrow$ Certolizumab+MTX provided 13.99 QALYs versus 13.92 QALYs for adalimumab+MTX $\rightarrow$ Rituximab+MTX $\rightarrow$ Sc Tocilizumab+MTX $\rightarrow$ Etanercept $+\mathrm{MTX} \rightarrow$ Certolizumab+MTX. In scenario 2 tofacitinib+MTX $\rightarrow$ Adalimumab+MTX $\rightarrow$ Rituximab+MTX $\rightarrow$ sc $\quad$ Tocilizumab $+\mathrm{MTX} \rightarrow$ Etanercept+MTX provided 13.75 QALYs versus 13.62 QALYs for Baricitinib+MTX $\rightarrow$ Adalimumab + MTX $\rightarrow$ Rituximab + MTX $\rightarrow$ Sc $\quad$ Tocilizumab + MTX $\rightarrow$ Etanercept+MTX

Tofacitinib-containing sequences provided lower total costs than the alternative sequences ( $€ 5,783$ and $€ 13,975$ for the pairwise comparisons previously described), resulting, therefore, dominant options versus sequences excluding tofacitinib.

On the probabilistic sensitivity analyses, sequences initiating with tofacitinib resulted cost-effective in $64.0 \%$ (scenario 1) and $56.9 \%$ (scenario 2) of the 1,000 iterations performed, because incremental cost-effectiveness ratio fell below a $€ 25,000 / Q A L Y$ gained willingness to pay threshold.

Conclusion: These results suggest that the inclusion of tofacitinib could be a dominant strategy for treatment of moderate to severe RA patients after DMARDs failure in Spain.

Disclosure of Interests: Carmen Peral Shareholder of: Carmen Peral is employee of and shareholder in Pfizer, Employee of: Carmen Peral is employee of and shareholder in Pfizer, Alejandro Balsa Grant/research support from: Abbvie, Pfizer, Novartis, BMS, Nordic, Sanofi, Consultant for: Abbvie, Pfizer, Novartis, BMS, Nordic, Sanofi, Sandoz, Lilly, Paid instructor for: Pfizer, Speakers bureau: Pfizer, Novartis, UCB, Nordic Sanofi, Sandoz, Lilly, Federico Navarro Grant/research support from: Pfizer, Consultant for: Pfizer, José Luis Pablos: None declared, Jose Manuel Martinez Sesmero Consultant for: Pfizer, Susana Gómez Employee of: I am a current employee of Pfizer., Monica Valderrama Shareholder of: Have received shares from Pfizer, Employee of: employee in Pfizer, Itziar Oyagüez Consultant for: I am employee of PORIB a consultant company which has received financial support from Grünenthal for development of this project, Miguel Angel Casado Grant/research support from: Pfizer, Consultant for: Pfizer, Fernando De Andres Grant/research support from: Pfizer, Employee of: Pfizer, Maria Montoro Shareholder of: Maria Montoro is employee of and shareholder in Pfizer, Employee of: Maria Montoro is employee of and shareholder in Pfizer DOI: 10.1136/annrheumdis-2019-eular.6741

\section{THU0188 INHIBITION OF STRUCTURAL JOINT DAMAGE WITH UPADACITINIB AS MONOTHERAPY OR IN COMBINATION WITH METHOTREXATE IN PATIENTS WITH RHEUMATOID ARTHRITIS: 1 YEAR OUTCOMES FROM THE SELECT PHASE 3 PROGRAM}

Charles Peterfy ${ }^{1}$, Mark C. Genovese ${ }^{2}$, In-Ho Song ${ }^{3}$, Alan Friedman ${ }^{3}$

Stephen Hall ${ }^{4}$, Eduardo Mysler ${ }^{5}$, Patrick Durez ${ }^{6}$, Xenofon Baraliakos ${ }^{7}$, Jose Jeffrey Enejosa ${ }^{3}$, Tim Shaw $^{3}$, LI Yihan ${ }^{3}$, Su Chen ${ }^{3}$, Vibeke Strand ${ }^{2}$. ${ }^{1}$ Spire Sciences, Inc., Kentfield, United States of America; ${ }^{2}$ Stanford University, Palo Alto, United States of America; ${ }^{3}$ AbbVie, Inc., North Chicago, United States of America; ${ }^{4}$ Monash University, Cabrini Health and Emeritus Research, Malvern, Australia ${ }^{5}$ Organización Médica de Investigación, Buenos Aires, Argentina; ${ }^{6}$ Cliniques Universitaires Saint-Luc, Université Catholique de Louvain, Brussels, Belgium; ${ }^{7}$ Rheumazentrum Ruhrgebiet, Herne and Ruhr-University Bochum, Bochum, Germany

Background: Long-term prevention of structural joint damage is a key treatment goal in the management of $R^{1}$. Upadacitinib (UPA), a JAK1selective inhibitor, inhibited the progression of structural joint damage at 6 months as monotherapy in methotrexate (MTX)-naïve RA patients (pts) ${ }^{2}$ and in combination with MTX in pts with inadequate response (IR) to $\mathrm{MTX}^{3}$.

Objectives: To evaluate the progression of structural joint damage (radio graphic) through Week 48 in pts with moderately to severely active RA treated with UPA monotherapy or in combination with MTX.

Methods: Radiographic progression was assessed in 2 phase 3 randomized controlled trials (RCTs), as previously described ${ }^{2,3}$. MTX-naïve pts were randomized to UPA 15 or $30 \mathrm{mg}$ QD or MTX monotherapy [SELECT-EARLY, $\mathrm{N}=945$ ], while MTX-IR pts were randomized to UPA $15 \mathrm{mg}$ QD or adalimumab (ADA) $40 \mathrm{mg}$ eow or placebo (PBO), with continuous background MTX [SELECT-COMPARE, $\mathrm{N}=1629$ ]. Both RCTs specifically enrolled pts at high risk for progression of joint damage (high disease activity including elevated hsCRP, presence of baseline erosions and ACPA and/or RF positivity ${ }^{2,3}$ ). The mean changes $(\Delta)$ from baseline 